\title{
Flt3/MerTK Inhibitor MRX-2843
}

National Cancer Institute

\section{Source}

National Cancer Institute. Flt3/MerTK Inhibitor MRX-2843. NCI Thesaurus. Code

C151407.

An orally bioavailable inhibitor of two receptor tyrosine kinases (RT Ks), FMS-like tyrosine kinase-3 (Flt3; CD135; fetal liver kinase-2; Flk2) and tyrosine-protein kinase Mer (MerTK; proto-oncogene c-Mer; Mer), with potential antineoplastic activity. Upon administration, MRX-2843 targ ets and binds to both Flt3 and MerTK. This prevents lig and-dependent phosphorylation and activation of Flt3 and MerTK, which inhibits the activation of their downstream signaling pathways. This induces apoptosis and inhibits proliferation of Flt3and/or MerT K-overexpressing tumor cells. Flt3 and MerTK, are overexpressed in certain tumor cell types and play key roles in tumor cell proliferation and survival. 